\title{
ON HOLOMORPHIC GRADED MANIFOLDS
}

\author{
PAUL GREEN ${ }^{1}$
}

\begin{abstract}
Kostant's definition of a $C^{\infty}$-graded manifold is adapted to the holomorphic category. It is shown that, in contrast to the $C^{\infty}$ case, there exist holomorphic graded manifolds with structure sheaf not isomorphic to the sheaf of sections of a bundle of exterior algebras.
\end{abstract}

Kostant [2] has given a definition of a graded manifold as a pair $(X, A)$ where $X$ is a $C^{\infty}$-manifold and $A$ is a sheaf of graded-commutative algebras over the real numbers, which locally can be given the structure of a finite-dimensional exterior algebra over the sheaf of $C^{\infty}$-functions on $X$, in such a way that the augmentation is globally defined.

Batchelor [1] has proven that if $(X, A)$ is a graded manifold in Kostant's sense, then $A$ is isomorphic to the sheaf of sections of the bundle of exterior algebras associated to a vector bundle. Our purpose here is to show that Batchelor's theorem does not carry over to the natural adaptation of Kostant's definition to the holomorphic category.

We will rely on the definition of a graded-commutative algebra given in [2], except that our algebras are over the complex numbers and may be either $Z$-graded or $Z_{2}$ graded. We will write $Z$-gradations as superscripts and $Z_{2}$-gradations as subscripts.

$X$ denotes a complex analytic manifold with structure sheaf $O_{X}$. We define an augmented sheaf of $Z_{2}$-graded commutative algebras over $X$ to be a pair $(A, \alpha)$ such that $A$ is a sheaf of $Z_{2}$-graded-commutative algebras, $\alpha: A \rightarrow O_{X}$ is a locally split epimorphism of sheaves of algebras, and $I_{\alpha}=\operatorname{Ker} \alpha$ is the ideal sheaf of $A$ generated by $A_{1}$. Homomorphisms of augmented sheaves of $Z_{2}$-graded-commutative algebras are simply homomorphisms of sheaves of $Z_{2}$-graded algebras, since the kernel of the augmentation is preserved along with the gradation and $O_{X}$ has no nontrivial automorphisms as a sheaf of complex algebras. In the sequel, we will write "augmented sheaf" for "augmented sheaf of $Z_{2}$-graded-commutative algebras."

A sheaf, $\Lambda$, of $Z$-graded-commutative algebras is called connected if $\Lambda^{0}=0_{X}$. We will refer to such a sheaf simply as a connected sheaf. Homomorphisms of connected sheaves are homomorphisms of sheaves of $Z$-graded algebras.

If $(A, \alpha)$ is an augmented sheaf, we define $\tilde{A}^{k}=I_{\alpha}^{k} / I_{\alpha}^{k+1} ; k>0, \tilde{A}^{0}=0_{X}$. Then $\tilde{A}$ is a connected sheaf with product induced from the product in $A$. This construction is evidently functorial, and we write $\tilde{f}$ for the homomorphism of connected sheaves induced by a homomorphism, $f$, of augmented sheaves.

Received by the editors September 24, 1981 and, in revised form, November 2, 1981.

1980 Mathematics Subject Classification. Primary 32C35.

${ }^{1}$ The author wishes to thank Wolfson College and the Mathematical Institute, Oxford, whose kind hospitality facilitated the research for this paper. 
Let $E$ denote (now and throughout the sequel) a holomorphic vector bundle over $X$. We will write $\Lambda E$ for the connected sheaf of germs of sections of the bundle of exterior algebras on $E$. We define an augmented sheaf $\left(A_{E}, \alpha_{E}\right)$ by

$$
\left(A_{E}\right)_{0}=\sum_{k \text { even }} \Lambda^{k} E \text { and }\left(A_{E}\right)_{1}=\sum_{k \text { odd }}\left(\Lambda^{k} E\right)
$$

where $\alpha_{E}$ is the natural projection on $\Lambda_{0} E=O_{X}$. There is a canonical identification of $\tilde{A}_{E}$ with $\Lambda E$.

We say that an augmented sheaf $(A, \alpha)$ defines a holomorphic graded manifold structure on $X$ if, for some $E, \tilde{A}$ is isomorphic to $\Lambda E$ as a connected sheaf.

Proposition 1. Let $(A, \alpha)$ be an augmented sheaf, and let $\theta: \Lambda E \rightarrow \tilde{A}$ be an isomorphism of connected sheaves. Then $X$ is covered by open sets, $U$, such that there exist isomorphisms $f_{U}: A_{E}|\mathcal{U} \rightarrow A| \mathcal{U}$ such that $\tilde{f}_{\mathcal{U}}=\theta \mid \mathcal{U}$.

Proof. Let $x \in X$, and let $\mathcal{U}$ be a neighborhood of $x$ such that $E \mid \mathcal{U}$ is trivial and $\alpha \mid \mathcal{U}$ is split. Let $c: O_{U} \rightarrow A_{0} \mid \mathcal{U}$ be a splitting of $\alpha$. Let $e_{1}, \ldots, e_{n}$ be a basis for $\Lambda^{1} E \mid \mathcal{U}$ over $O_{u}$.

Let $\tilde{a}_{i} \in \tilde{A}^{1}(U)=\theta\left(e_{i}\right)$ for $1 \leq i \leq n$. Then (making $U$ smaller if necessary) we may choose $a_{i} \in A_{1}(U)$ with $a_{i}+I_{\alpha}=\tilde{a}_{i}, 1 \leq i \leq n$. If we write $f_{u} \mid O_{u}=c$; $f_{u}\left(e_{i}\right)=a_{i}, 1 \leq i \leq n$, fu extends uniquely to a homomorphism of connected sheaves from $\Lambda E \mid U$ to $\tilde{A} \mid \mathcal{U}$. It is evident that $\tilde{f}_{\mathcal{U}}=\theta \mid \mathcal{U}$, from which it follows that $f_{u}$ is an isomorphism.

Let $\operatorname{Aut}(E)$ denote the automorphism sheaf of $E$ as a vector bundle. Then $\operatorname{Aut}(E)$ is also the automorphism sheaf of $\Lambda E$ as a connected sheaf. Aut $(E)$ is thus a subsheaf of $\operatorname{Aut}\left(A_{E}\right)$, the automorphism sheaf of the augmented sheaf $\left(A_{E}, \alpha_{E}\right)$, since $A_{E}$ and $\Lambda E$ have the same underlying sheaf of algebras, and any automorphism which preserves the $Z$-grading a fortiori preserves the $Z_{2}$-grading. If we write $j$ for the inclusion of $\operatorname{Aut}(E)$ in $\operatorname{Aut}\left(A_{E}\right)$, and define $G_{E}$ as a subsheaf of $\operatorname{Aut}\left(A_{E}\right)$ by $f \in G_{E}$ iff $\tilde{f}: \Lambda E \rightarrow \Lambda E$ is the identity, we have the right split exact sequence of sheaves of groups

$$
1 \rightarrow G_{E} \rightarrow \operatorname{Aut}(\underbrace{\left.A_{E}\right) \underset{\sim}{\sim} \operatorname{Aut}(E)}_{j} \rightarrow 1 .
$$

Since $G_{E}$ is not in general a sheaf of Abelian groups, $H^{1}\left(X, G_{E}\right)$ has the structure only of a pointed set rather than a group. From the splitting, $H^{0}(X, \operatorname{Aut}(E))$ acts (by inner automorphisms of $\operatorname{Aut}\left(A_{E}\right)$ ) on $G_{E}$ and hence also on $H^{1}\left(X, G_{E}\right)$ in a base point preserving fashion.

PROPOSITION 2. The isomorphism classes of augmented sheaves $(A, \alpha)$, with $\tilde{A}$ isomorphic to $\Lambda E$, are parametrized by the orbits of $H^{1}\left(X, G_{E}\right)$ under the action of $H^{0}(X ;$ Aut $E)$ in such a way that the base point corresponds to $\left(A_{E}, \alpha_{E}\right)$.

Proof. Let $(A, \alpha)$ be an augmented sheaf, and let $\theta: \Lambda E \rightarrow \tilde{A}$ be an isomorphism of connected sheaves. Then by Proposition $1, X$ is covered by open sets $U$ admitting isomorphisms of augmented sheaves $f_{U}: A_{E}|\mathcal{U} \rightarrow A| \mathcal{U}$ with $\tilde{f}_{\mathcal{U}}=\theta \mid \mathcal{U}$. For $\mathcal{U} \cap \mathcal{V}$ nonempty, write $g u, v=f \bar{\nu}^{1} f u$. Then by standard arguments, $\{g u, v\}$ is a cocycle which defines a class $h(A, \alpha, \theta) \in H^{1}\left(X, G_{E}\right)$ which is independent of the choices of $U$ and $f U$ and satisfies the following.

(1) Any class in $H^{1}\left(X, G_{E}\right)$ is realized as $h(A, \alpha, \theta)$ for some $(A, \alpha, \theta)$. 
(2) $h(A, \alpha, \theta)=h(B, \beta, \varphi)$ if and only if there is an isomorphism $f:(A, \alpha) \rightarrow$ $(B, \beta)$ with $\varphi \circ \tilde{f}=\theta$.

(3) For $\chi \in H^{0}($ Aut $E), h(A, \alpha, \theta \circ \chi)=\chi(h(A, \alpha, \theta))$.

(4) $h\left(A_{E}, \alpha_{E}\right.$, id $)=$ the base point.

The proposition evidently follows.

Proposition 3. For any holomorphic vector bundle $E$ over $X, G_{E}$ has a decreasing filtration by normal subgroup sheaves $G_{E}^{k}$ with the following properties.

(i) $G_{E}^{2}=G_{E}$.

(ii) $G_{E}^{k} / G_{E}^{k+1} \approx \operatorname{Der}_{O_{X}}\left(O_{X}, \Lambda^{k} E\right)$, $k$ even.

(iii) $G_{E}^{k} / G_{E}^{k+1} \approx \operatorname{Hom}_{O_{x}}\left(\Lambda^{1} E, \Lambda^{k} E\right), k$ odd.

Proof. Write $i_{k}: \Lambda^{k} E \rightarrow A_{E}$ and $\pi_{k}: A_{E} \rightarrow \Lambda^{k} E$, respectively, for the projections and inclusions induced by the equality of $A_{E}$ and $\Lambda E$ as rings. We define $g$ to be in $G_{E}^{k}$ provided $g(x)-x \in I_{\alpha_{E}}^{k}$ for all $x \in A_{E}$. $G_{E}^{k}$ is normal in $G_{E}$ by the observation $h^{-1} g h(x)-x=h^{-1}(g h(x)-h(x)) . G_{E}^{2}=G_{E}$ by the condition (defining for $G_{E}$ ) that $\tilde{g}$ is the identity on $\Lambda E$.

For $g \in G_{E}^{k}, k$ even, we define $a_{k}(g)=\pi_{k} \circ g \circ i_{0}$. Using the observation that $\pi_{j+l} \circ g \circ i_{j}=0$ for $l<k$, it is easily verified that $a_{k}(g)$ is a derivation from $O_{X}=\Lambda^{0} E$ into $\Lambda^{k} E$, that $a_{k}\left(g_{1} g_{2}\right)=a_{k}\left(g_{1}\right)+a_{k}\left(g_{2}\right)$ and that $a_{k}(g)=0$ iff $g \in G_{E}^{k+1}$.

Similarly for $g \in G_{E}^{k}, k$ odd, we define $b_{k}(g)=\pi_{k} \circ g \circ i_{1}$ and verify that $b_{k}$ is a homomorphism from $G_{E}^{k}$ to $\operatorname{Hom}_{O_{x}}\left(\Lambda^{1} E, \Lambda^{k} E\right)$ whose kernel is $G_{E}^{k+1}$.

It remains to show that $a_{k}$ and $b_{k}$ are epic on sufficiently small open sets. For $k$ odd, any homomorphism $s$ from $\Lambda^{1} E$ to $\Lambda^{k} E$ extends uniquely to an $O_{X}$-linear derivation of $A_{E}$ which exponentiates to a gradation preserving automorphism $g \in$ $G_{E}^{k}$ with $b_{k}(g)=s$. In the even case we must first choose an $O_{X}$ basis of $\Lambda^{1} E$ on some sufficiently small open set. Then any derivation $d$ of $O_{X}$ into $\Lambda^{k} E$ extends uniquely to a derivation (not $O_{X}$-linear) of $A_{E}$ which annihilates the chosen basis. Once again we may now exponentiate to an automorphism $g \in G_{E}^{k}$ with $a_{k}(g)=d$. In each case the exponential power series terminates because $E$ is finite dimensional and $I_{\alpha_{E}}$ is nilpotent.

THEOREM I. Let $E$ be a holomorphic vector bundle of dimension 2 over $X$ with $H^{1}\left(T(X) \otimes \Lambda^{2} E\right) \neq 0$. Then there exists an augmented sheaf $(A, \alpha)$ over $X$ for which $\tilde{A}$ is isomorphic to $\Lambda E$ as a connected sheaf, but $A$ is not isomorphic to $A_{E}$ as an augmented sheaf.

ProOF. In this case $I_{E}^{3}=0$. Hence $G_{E}^{3}=1$ and $G_{E}=G_{E}^{2}=\left(T(X) \otimes \Lambda^{2} E\right)$ by Proposition 3 . Hence $H^{1}\left(X, G_{E}\right)$ is nontrivial and, therefore, has at least one orbit other than the base point under the action of $H^{0}(X$, Aut $E)$. The conclusion of the theorem now follows by Proposition 2.

REMARK. If $(A, \alpha)$ is as in the conclusion of Theorem I, it is not possible that $A$ is isomorphic to $A_{E^{\prime}}$ for some other bundle $E^{\prime}$, since in that case we would have $\Lambda E^{\prime} \approx \tilde{A}_{E^{\prime}} \approx \Lambda E$ as connected sheaves, from which it follows that $E^{\prime} \approx E$.

In particular, the existence of a counterexample to Batchelor's theorem follows from the existence of $X$ and $E$ satisfying the hypothesis of Theorem $\mathrm{I}$. The simplest example is $X=C P^{1} ; E=2 T^{*}\left(C P^{1}\right)$. It is then standard that $H^{1}\left(T(X) \otimes \Lambda^{2} E\right) \approx$ C. 


\section{REFERENCES}

1. M. Batchelor, The structure of supermanifolds, Trans. Amer. Math. Soc. 253 (1979), 329-388.

2. B. Kostant, Graded manifolds, graded Lie theory, and prequantization, Differential Geometrical Methods in Mathematical Physics, Lecture Notes in Math., vol. 570, Springer-Verlag, Berlin and New York, pp. 177-306.

Department of Mathematics, University of Maryland, College Park, MARYLAND 20742 